\section{SAT0448 BONE HEALTH IN PATIENTS WITH PSORIASIS ARTHRITIS IN THE SWISS NATIONAL COHORT: A CROSS-SECTIONAL STUDY}

I. Youssef ${ }^{1}$, B. Aubry-Rozier ${ }^{2}$, D. Aeberli ${ }^{3}$, D. Dan ${ }^{4} .{ }^{1}$ University of Lausanne, Lausanne, Switzerland; ${ }^{2}$ University Hospital Lausanne, Rheumatology and Center of Bone Diseases, Lausanne, Switzerland; ${ }^{3}$ University Hospital Bern, Rheumatology, Bern, Switzerland; ${ }^{4}$ University Hospital Lausanne, Rheumatology, Lausanne, Switzerland

Background: There is much controversy surrounding the loss of bone mass in patients with psoriasis arthritis (PsA).

Objectives: To evaluate the prevalence of osteoporosis (OP) and fracture in patients with PsA in the Swiss Clinical Quality Management (SCQM) cohort, a large national database of patients with inflammatory arthritis; to study different factors influencing bone health and the correlation between disease activity, treatment and occurrence of densitometric osteoporosis or fracture.

Methods: We analyzed all PsA-patients included in the cohort from 2006-April 2019. We evaluated demographic and clinical data: age, gender, BMI, disease duration, smoking/alcohol habits, patient's and physician's global assessment, joint count, $H A Q$, medication and inflammatory activity measured by ESR, CRP, DAS 28 and DAPSA score. We compared patients with BMD measurement (DXA) with the group without DXA (nDXA). In DXA group we analyzed patients according to osteoporotic status and did subgroup analysis in premenopausal, menopausal women and men.

Results: Of the 2443 patients, 545 had a DXA. Age of scanned patients was 18-84 years. Only 295 BMD data were available for analysis. DXA patients were 6.4 years older $(54.2 \pm 11,1$ vs $47.8 \pm 12.4$ years, $p<0.001)$, and were more female $(67 \%$ vs $43 \%)$. Duration of the disease was longer $(6.6 \pm 8.3$ vs $5.3 \pm 7.1 \mathrm{y}, \mathrm{p}<0.001)$ in DXA group. DAS28-CRP and DAS28-ESR were higher in DXA group $(3,1 \pm 1,2$ vs $2,9 \pm 1,1, p<0,04$ and $3,2 \pm 1,4$ vs $3 \pm 1,3, p<$ $0,002$, respectively), as was the DAPSA score (32 \pm 30 vs $27 \pm 20 p<0.04)$. Patients in DXA group were more exposed to prednisone and conventional DMARDs ( $15.4 \%$ vs $4.7 \%, p<0.001$ and $51.7 \%$ vs $43 \%, p<0.01$ respectively). There were more fractures in DXA- than in nDXA group $(5.7 \%$ vs $1.4 \%, \mathrm{P}<$ $0.001)$.

In DXA group $18.4 \%$ patients had OP and $50.2 \%$ osteopenia. Patient characteristics are shown in Table 1. We confirmed a positive correlation between femoral and lumbar BMD and BMI, and between higher age and lower femoral BMD. Disease duration was inversely correlated with femoral, but not lumbar BMD. Interestingly, other variables, including disease activity, showed no significant correlation with the BMD, but OP patients had higher disease activity.

Table 1. Comparison between patients with and without OP in DXA group. *: statistically significant. Values are expressed as mean and standard deviation unless stated otherwise.

\begin{tabular}{lccc}
\hline & $\begin{array}{c}\text { NO OSTEOPOROSIS } \\
(\mathrm{n}=244)\end{array}$ & $\begin{array}{c}\text { OSTEOPOROSIS } \\
(\mathrm{n}=51)\end{array}$ & $\mathrm{P}$ \\
\hline Age & $52.9 \pm 9,8$ & $56.3 \pm 13,4$ & $0,04^{*}$ \\
Female \% & 67.6 & 68.6 & 0,88 \\
Disease duration (y) & $6,3 \pm 8$ & $7,7 \pm 9,9$ & 0,339 \\
BMI & $26,6 \pm 5$ & $22,7 \pm 4,8$ & $0,0001^{*}$ \\
Smoking \% & 19,9 & 29 & 0,29 \\
Alcohol \% & 77,2 & 68,6 & 0,37 \\
HAQ & $0,7 \pm 0,5$ & $0,9 \pm 0,7$ & $0,03^{*}$ \\
PGA & $3,8 \pm 2,2$ & $4,1 \pm 2,5$ & 0,329 \\
ESR & $16,6 \pm 16,7$ & $20,8 \pm 20,9$ & 0,2 \\
CRP & $9,1 \pm 12,8$ & $16,9 \pm 36,2$ & $0,01^{*}$ \\
DAS28_ESR & $3,2 \pm 1,4$ & $3,6 \pm 1,3$ & 0,08 \\
DAS28_CRP & $3,1 \pm 1,2$ & $3,4 \pm 0,9$ & $0,04^{*}$ \\
DAPSA & $29,6 \pm 23,6$ & $37,8 \pm 43,5$ & $0,04^{*}$ \\
Fracture \% & 2 & 3,9 & 0,4 \\
Anti-TNF\% & 35,7 & 25,5 & 0,16 \\
csDMARD \% & 53,7 & 54,5 & 0,6 \\
tsDMARD \% & 0,4 & 2 & 0,22 \\
Prednisone \% & 15,2 & 13,7 & 0,069 \\
\hline
\end{tabular}

Subgroup analysis (Figure 1) showed higher OP prevalence in postmenopausal women $(22,6 \%)$ vs in men $(16.8 \%)$ or premenopausal women $(10.7 \%)$ in concordance with the fracture rate $(8.3 \%$ vs $6.3 \%$ vs $3.9 \%)$. BMD was lower and the disease activity was higher in postmenopausal women compared to the others groups.

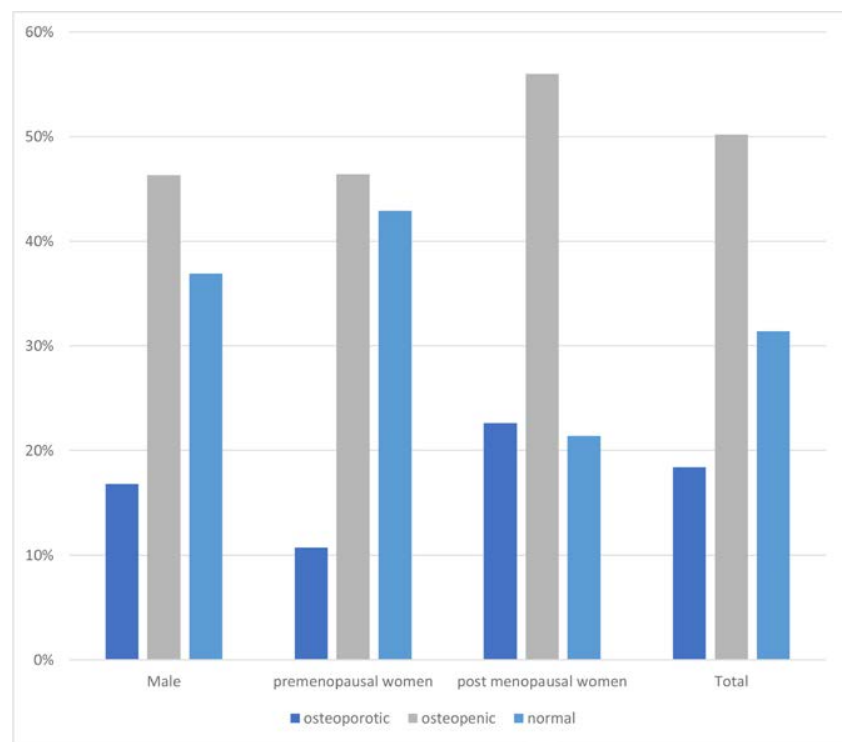

Figure 1. Subgroup analysis on the prevalence of OP and osteopenia

Conclusion: Our data suggest that Swiss clinician are aware of risk of poor bone-health in PsA patients and perform DXA in this population even in younger patients and in men. Interestingly, we describe that the patients with OP had higher disease activity and poorer functional status than patients without OP. Longitudinal studies are needed to evaluate bone quality, fractures, and relationship between bone health in PsA and disease associated factors. They should integrate parameters of bone turnover and use an appropriate control group.

Disclosure of Interests: None declared

DOI: 10.1136/annrheumdis-2020-eular.2629

\section{SAT0449 \\ SPONDYLOARTHRITIS AND FRACTURE RISK: DOES DXA REALLY HAVE AN IMPACT IN THE RISK OF FRACTURE ESTIMATED BY FRAX?}

B. M. Fernandes ${ }^{1}$, S. Garcia ${ }^{1}$, S. Ganhão ${ }^{1}$, M. Rato ${ }^{1}$, F. Pinheiro', M. Bernardes ${ }^{1}$, L. Costa ${ }^{1} .{ }^{1}$ Centro Hospitalar Universitário São João, Rheumatology, Porto, Portugal

Background: Low bone mineral density (BMD) is common in ankylosing spondylitis (AS). The fracture risk (FR) is increased and its reduction with pharmacologic therapy is not clearly defined in this population. However, early screening and bisphosphonates as first-line treatment are recommended.

Objectives: To investigate the influence of dual-energy X-ray absorptiometry (DXA) in the ten-year risk of fracture assessed by FR Assessment Tool (FRAX) and to determine possible demographic or clinical factors associated with an increased FR in a spondyloarthritis ( $\mathrm{SpA}$ ) population.

Methods: Retrospective study including all the over 40 years-old SpA patients (ASAS classification criteria) followed at our Rheumatology Department and registered in the national database. Demographic, clinical and laboratorial data were collected at the time of the last follow-up visit. Data from the last DXA (until 3 years prior to the last visit) were collected. Indication for pharmacological treatment by FRAX was assessed according to the national recommendations. Results: A total of $231 \mathrm{SpA}$ patients were included: 126 males (54.5\%), 53 (22.9\%) smokers; 171 (74\%) had AS, 23 (10\%) had Inflammatory Bowel Disease Associated SpA and $37(16 \%)$ had Undifferentiated SpA. At the last follow-up visit, the mean age was 52.9 years $( \pm 9.6)$ and the median disease duration was 21.9 years [1.0-55.5]. The mean ASDAS-CRP was $2.5( \pm 0.9)$ and the majority of patients had moderate $(25.5 \%)$ or high $(48.5 \%)$ disease activity (according to ASDAS). One hundred and thirty patients (56.3\%) were taking NSAIDs, 45 $(19.5 \%)$ were taking glucocorticoids, 85 (36.8\%) were under csDMARDs and $170(73.6 \%)$ under bDMARDs [157 (68\%) under TNFi, 11 (4.8\%) under secukinumab and $2(0.9 \%)$ under ustekinumab].

Eleven patients (4.8\%) had previous fragility fractures, $118(51.1 \%)$ had DXA in the last 3 years and 167 (72.3\%) were taking calcium and/or vitamin D supplements. Sixteen patients $(6.9 \%)$ had indication for treatment by FRAX without DXA and 9 of these $(56.3 \%)$ were already under treatment. Similarly, $16(6.9 \%)$ had indication for treatment by FRAX with DXA and 13 of these $(81.3 \%)$ were already under treatment. Ten patients (4.3\%) were reclassified in FRAX with DXA: 7 (3\%) had no indication for treatment by FRAX without DXA but obtained it by FRAX with 